\section{Desigualdades regionales en la mortalidad por diabetes mellitus y en el acceso a la salud en Argentina}

\author{
Regional inequalities in mortality from diabetes \\ mellitus and access to health in Argentina
}

\author{
Desigualdades regionais na mortalidade por
diabetes mellitus e no acesso à saúde \\ na Argentina
}

María Jimena Marro 1

Andrey Moreira Cardoso 2

Iuri da Costa Leite 2

doi: 10.1590/0102-311X00113016

\section{Resumen}

El objetivo del estudio fue estimar la carga de mortalidad por grandes grupos de causas, con foco en la diabetes mellitus, así como sus diferenciales, según la presencia o ausencia de cobertura por algún sistema de salud, para Argentina y sus regiones. El estudio fue realizado en base a los óbitos de individuos de 30 años y más, ocurridos en el trienio 2009-2011, estratificados según sexo, franja etaria, región, causa básica de muerte y cobertura de salud. Fueron calculadas tasas de mortalidad y de años de vida perdidos por muerte prematura (AVPMP). La calidad de los registros de mortalidad de Argentina fue insatisfactoria, a expensas de la elevada proporción de códigos de causas de muerte poco útiles. Los principales resultados evidenciaron la preponderancia del grupo de enfermedades crónicas en la carga de mortalidad en todas las regiones del país. En el Noreste, una de las regiones más pobres del país, la carga de mortalidad fue elevada para todos los grupos de causas. Las tasas de mortalidad y de AVPMP por diabetes mellitus ajustadas fueron más elevadas en la región de Cuyo. Se destaca la diferencia marcada en las tasas ajustadas de AVPMP, según la situación de cobertura de salud. Este estudio orienta hacia el desarrollo de acciones tendientes a disminuir las desigualdades regionales $y$ socioeconómicas evidenciadas en los diferenciales de mortalidad en Argentina.

Diabetes Mellitus; Disparidades en el Estado de Salud; Mortalidad

\section{Correspondencia}

M. J. Marro

Instituto Nacional de Epidemiología Dr. Juan H. Jara. Ituzaingó 3520, Mar del Plata / Buenos Aires - 7600, Argentina.

jimenamarro@gmail.com

1 Instituto Nacional de Epidemiología Dr. Juan H. Jara, Mar del Plata, Argentina.

2 Escola Nacional de Saúde Pública Sergio Arouca, Fundação Oswaldo Cruz, Rio de Janeiro, Brasil. 
La diabetes mellitus forma parte de las enfermedades crónicas no transmisibles (ECNT). Constituye un problema de salud pública de relevancia mundial, con elevado costo humano, social y económico 1,2,3. Las tasas de incidencia y prevalencia de diabetes mellitus se encuentran en ascenso en países con diferentes níveles de ingresos en todo el mundo, a pesar de las variadas herramientas existentes para lidiar con la enfermedad.

La preponderancia de las ECNT se encuadra en un proceso denominado de transición demográfica, epidemiológica y nutricional, caracterizado por un descenso de la fecundidad, un desplazamiento de la mortalidad a grupos de edad más avanzada, con predominio de causas de muerte relacionadas con ECNT y un escenario alimentario con tendencia creciente al consumo de alimentos ricos en lípidos y azúcares, favorecedores del desarrollo de sobrepeso y obesidad en las poblaciones 4,5,6,7,8.

La prevalencia de diabetes mellitus en Argentina muestra una tendencia al aumento, variando de 8,4\% en al año 2005 a 9,8\% en 2013 9,10. Fue la séptima causa de muerte en el país en 2014, con 8.201 defunciones en ambos sexos y una tasa cruda de mortalidad de 19,2 por 100.000 habitantes 11.

A pesar de su importancia como indicador de salud, las tasas de mortalidad por causas no consiguen expresar las pérdidas sociales de esos óbitos. Un indicador con esa característica es el de años potenciales de vida perdidos (APVP) 12. Sin embargo, por no expresar las pérdidas en edades más avanzadas, los APVP han sido muy criticados. Desde el estudio fundacional, elaborado por Murray \& López ${ }^{13}$, el indicador de años de vida perdidos por muerte prematura (AVPMP), componente de mortalidad del estudio de carga de enfermedad, se convirtió en el más apropiado para medir el impacto de la mortalidad, incorporando el concepto de pérdida social.

Existen variados indicios de que la distribución de los resultados de salud, enfermedad y muerte se produce de manera desigual en las poblaciones 14,15,16,17. Las desigualdades, entendidas como variaciones cuantitativas en poblaciones expresadas a través de indicadores epidemiológicos, como evidencia empírica de tales diferencias 18, denotan una distribución subyacente injusta de los determinantes sociales de la salud, como acceso a educación, trabajo y cuidado de la salud 19.

Los resultados en la mortalidad por diabetes mellitus están íntimamente ligados a las condiciones de acceso a los servicios de salud en las poblaciones. Al tratarse de una enfermedad crónica, su evolución y pronóstico dependen de las opciones de diagnóstico oportuno, tratamiento adecuado y acceso a programas de cuidado 2,20 .

El objetivo de este estudio fue estimar la carga de mortalidad por grandes grupos de causas y diabetes mellitus en particular a nivel subnacional, así como valorar su distribución, según la condición de cobertura de salud, a fin de contribuir a la identificación de desigualdades en la situación de las enfermedades crónicas en Argentina, con énfasis en el peso de las muertes por diabetes.

\section{Métodos}

Se utilizó un diseño descriptivo de carga de mortalidad para Argentina, con nivel de agregación subnacional. Las unidades de análisis fueron las regiones estadísticas de la República Argentina, de acuerdo al agrupamiento adoptado por la Encuesta Nacional de Factores de Riesgo (ENFR) 2009 9: Gran Buenos Aires (GBA), Pampeana, Noroeste, Noreste, Cuyo y Patagónica.

La población bajo estudio correspondió a la de 30 años y más de la República Argentina para el año 2010. Los indicadores fueron construidos a partir de la media de los datos de mortalidad correspondientes al trienio 2009-2011.

Se utilizaron fuentes de datos secundarias: bases de mortalidad de los años 2009 a 2011, otorgadas por la Dirección de Estadísticas e Información en Salud (DEIS), del Ministerio de Salud de la Nación; y el Censo Nacional de Población y Viviendas de 2010 21, del Instituto Nacional de Estadísticas y Censos (INDEC).

De las bases de mortalidad, fueron utilizadas las variables sexo, franja etaria (30-44, 45-59, 60-69, 70-79, 80 o más años), provincia de residencia (variable utilizada para construir la regionalización, de acuerdo a la adoptada por la ENFR), causa básica de defunción, según la Clasificación Estadística Internacional de Enfermedades y Problemas Relacionados con la Salud - décima revisión (CIE-10) 22, cobertura de salud (afiliación a algún sistema de atención de la salud - obra social, plan privado o programa estatal de salud). 
Cabe la siguiente aclaración, en relación con las categorías de cobertura de salud en Argentina: obra social se refiere a organizaciones de cobertura de atención médica, dirigidas por trabajadores, con relación estrecha con los sindicatos, que funcionan como planes de salud corporativos, pertenecientes a categorías profesionales. El principio de solidaridad grupal es característico de las obras sociales, que no poseen fines de lucro 23; las prestaciones se subsidian a través de aportes y contribuciones de los afiliados. En cambio, los planes privados son organizados por grupos económicos corporativos que ofrecen servicios privados con fines lucrativos. Plan estatal de salud hace referencia al derecho a la atención de la salud que surge a partir del empadronamiento bajo algún programa estatal de salud, sea municipal, provincial o nacional. Si bien variable entre provincias, este último grupo representa un porcentaje pequeño de la población con cobertura de salud. Debido a que la gran mayoría de la población con cobertura utiliza habitualmente para su atención instituciones de salud privadas, tener cobertura de salud podría considerarse un proxy de cobertura proveniente de la red privada de salud.

Las muertes fueron clasificadas de acuerdo a la codificación de la causa básica de defunción en tres categorías: Grupo I - las enfermedades transmisibles, maternas, perinatales y nutricionales; Grupo II - las no transmisibles; y el Grupo III - las lesiones 24. Se focalizó en el Grupo II correspondiente a las enfermedades crónicas y especialmente en la diabetes mellitus (códigos CIE-10: E10-E14).

La calidad de las bases de datos de mortalidad fue verificada por las proporciones de óbitos por causas mal definidas, de óbitos con causa básica clasificada como código basura, y del total de causas poco útiles. Las causas mal definidas correspondieron al Capítulo XVIII, códigos CIE-10 R00-R99 22. Los códigos basura, que corresponden a causas de muerte poco útiles desde una perspectiva de políticas sanitarias, fueron definidos de acuerdo con la propuesta de Mathers et al. 25: códigos basura Grupo I (lesiones) - CIE-10 Y10-Y34 e Y872; códigos basura Grupo II (cardiovasculares) - CIE-10 I47.2, I49.0, I46, I50, I51.4, I51.5, I51.6, I51.9, I70.9; y códigos basura Grupo III (cáncer) - CIE-10 C76, C80 y C97. El total de causas poco útiles resultó de la suma de los tres grupos de códigos basura y las causas mal definidas.

No se utilizaron técnicas de corrección del subregistro de muerte, debido a que el subregistro de mortalidad en Argentina fue estimado en un 1,4\% para el año 201126.

De manera similar al estudio de carga de enfermedad, realizado en Brasil en 1998, se realizó una redistribución de los registros con datos faltantes para sexo, franja etaria, región, cobertura de salud, de manera proporcional a la distribución conocida de frecuencias de las categorías de la variable en cuestión, incluyendo las causas de muerte con tres dígitos. Las causas mal definidas fueron redistribuidas también proporcionalmente, de acuerdo a la distribución conocida de causas para el mismo año, sexo, región, franja etaria y cobertura de salud 27.

Fueron estimadas las proporciones de mortalidad por grupos de causas y para diabetes mellitus.

Las tasas específicas de mortalidad para 2010, según sexo, región, grupos de causa (Grupos I, II y III) y diabetes mellitus se calcularon utilizando como numerador la media anual de muertes en el periodo 2009-2011, dividido por la población en riesgo estimada al 10 de julio de 2010. Finalmente, se estimaron los números absolutos y tasas de AVPMP según sexo, región, grupos de causa, diabetes mellitus y cobertura de salud.

Los AVPMP para determinada causa de muerte se calcularon como el producto entre el número de muertes, debido a esa causa, sexo y franja etaria, y la esperanza de vida, estimada para ese sexo y franja etaria 28, de acuerdo a la tabla de vida teórica para Argentina.

La tabla de vida teórica se calculó utilizando las tasas más bajas de mortalidad por franja etaria, según el sexo y la región. La tabla de vida teórica propuesta para Argentina, según sexo, se presenta en la Tabla 1. Con el objeto de su construcción, se siguieron los pasos propuestos por el Institute for Health Metrics and Evaluation (IHME; Seatlle, EE.UU.) en el ejercicio de construcción de la tabla de vida 29.

En todos los casos en que se realizó ajuste de tasas, se tomó como población estándar a la de la República Argentina ,según franja etaria y sexo, para el año 201021.

Fue construido un intervalo de confianza del $95 \%$ para las tasas ajustadas.

Se adoptaron las últimas recomendaciones de expertos de no utilizar la tasa de descuento, ni la función de ponderación de la edad 24,30.

Se utilizaron los paquetes estadísticos: Microsoft Excel 2007 (Microsoft Corp., EE.UU.); R versión 3.1.0, paquete "Epitools" para la estandarización de tasas (The R Foundation for Statistical Computing, Viena, Austria); y SAS versión 9.3 (SAS Inst., Cary, EE.UU.), para la redistribución de campos vacíos. 
Tabla 1

Tabla de vida para Argentina según sexo, utilizada en la investigación, 2010 (tabla de vida teórica).

\begin{tabular}{|c|c|c|}
\hline \multirow[t]{2}{*}{ Edad (años) } & \multicolumn{2}{|c|}{ Esperanza de vida } \\
\hline & Varones & Mujeres \\
\hline$<1$ & 73,6 & 80,1 \\
\hline $1-4$ & 73,4 & 79,8 \\
\hline $5-9$ & 69,5 & 75,9 \\
\hline $10-14$ & 64,6 & 71,0 \\
\hline $15-19$ & 59,7 & 66,0 \\
\hline $20-24$ & 55,0 & 61,1 \\
\hline $25-29$ & 50,3 & 56,3 \\
\hline $30-34$ & 45,7 & 51,4 \\
\hline $35-39$ & 41,0 & 46,6 \\
\hline $40-44$ & 36,3 & 41,8 \\
\hline $45-49$ & 31,7 & 37,1 \\
\hline $50-54$ & 27,2 & 32,4 \\
\hline $55-59$ & 23,0 & 28,0 \\
\hline $60-64$ & 19,1 & 23,7 \\
\hline $65-69$ & 15,6 & 19,6 \\
\hline $70-74$ & 12,4 & 15,7 \\
\hline $75-79$ & 9,7 & 12,2 \\
\hline $80-84$ & 7,4 & 9,1 \\
\hline $85-89$ & 5,5 & 6,5 \\
\hline $90-94$ & 4,3 & 4,7 \\
\hline $95-99$ & 2,3 & 2,5 \\
\hline$\geq 100$ & 2,3 & 2,5 \\
\hline
\end{tabular}

\section{Aspectos éticos}

La investigación respetó las consideraciones éticas previstas por la Guía de Investigación en Salud Humana del Ministerio de Salud de la Nación 31 y fue aprobada por el comité de ética de investigación del Instituto Nacional de Epidemiología Dr. Juan H. Jara (Mar del Plata, Argentina). No se evidenciaron conflictos de intereses.

\section{Resultados}

El análisis de la calidad de registros en la base completa de defunciones para el trienio 2009-2011 develó una proporción total de causas poco útiles en Argentina del 21,1\% (DE: 5,1), siendo las proporciones de causas mal definidas, códigos basura grupo lesiones, cardiovasculares y cáncer, de 8,5\%; $0,9 \% ; 10,4 \%$ y $1,3 \%$, respectivamente.

La proporción de causas mal definidas varió de 1,8\% en Cuyo, a 12,6\% en la región Pampeana. La proporción de códigos basura grupo cardiovasculares varió de 5\% en las regiones Noreste y Patagónica a $16 \%$ en GBA. Hubo un 4,2\% de códigos basura grupo lesiones en un conjunto de defunciones cuya región de residencia fue ignorada (Tabla 2).

Hubo 292.425 muertes de personas de 30 años o más, lo que representa el 93,1\% del total de óbitos registrado para el trienio 2009-2011. La proporción de defunciones fue similar en ambos sexos (varones: 51,1\%; mujeres 48,9\%). La proporción de muertes aumentó con la edad, alcanzando $41 \%$ en el grupo de 80 años y más. Las regiones Pampeana y GBA concentraron más del 70\% de las defunciones del país. 


\section{Tabla 2}

Proporción anual de causas mal definidas, códigos basura y causas poco útiles en la base completa de defunciones.

Argentina y regiones, 2009-2011.

\begin{tabular}{|c|c|c|c|c|}
\hline Indicadores & 2009 & 2010 & 2011 & Trienio \\
\hline \multicolumn{5}{|c|}{$\%$ causas mal definidas (signos y síntomas) * } \\
\hline Argentina & 8,6 & 8,4 & 8,4 & 8,5 \\
\hline GBA & 5,0 & 5,0 & 4,6 & 4,9 \\
\hline Pampeana & 12,1 & 12,5 & 13,2 & 12,6 \\
\hline Noroeste & 11,5 & 8,4 & 8,1 & 9,3 \\
\hline Noreste & 12,1 & 11,3 & 9,5 & 10,9 \\
\hline Cuyo & 1,9 & 1,8 & 1,8 & 1,8 \\
\hline Patagónica & 6,7 & 7,0 & 6,9 & 6,9 \\
\hline Ignorada & 7,1 & 7,1 & 7,4 & 7,3 \\
\hline \multicolumn{5}{|c|}{ \% códigos-basura (lesiones) * } \\
\hline Argentina & 0,9 & 0,9 & 1,0 & 0,9 \\
\hline GBA & 1,2 & 0,9 & 1,1 & 1,1 \\
\hline Pampeana & 0,9 & 1,0 & 1,1 & 1,0 \\
\hline Noroeste & 0,7 & 0,8 & 0,9 & 0,8 \\
\hline Noreste & 0,5 & 0,8 & 0,9 & 0,7 \\
\hline Cuyo & 0,1 & 0,1 & 0,1 & 0,1 \\
\hline Patagónica & 0,8 & 0,6 & 0,6 & 0,7 \\
\hline Ignorada & 4,1 & 4,7 & 3,8 & 4,2 \\
\hline \multicolumn{5}{|c|}{ \% códigos-basura (cardiovasculares) * } \\
\hline Argentina & 10,0 & 10,5 & 10,5 & 10,4 \\
\hline GBA & 15,9 & 16,4 & 16,1 & 16,1 \\
\hline Pampeana & 7,6 & 8,2 & 8,5 & 8,1 \\
\hline Noroeste & 4,9 & 6,0 & 5,9 & 5,6 \\
\hline Noreste & 4,4 & 5,4 & 5,1 & 5,0 \\
\hline Cuyo & 6,4 & 6,7 & 6,7 & 6,6 \\
\hline Patagónica & 4,7 & 4,8 & 5,4 & 5,0 \\
\hline Ignorada & 7,7 & 10,4 & 8,9 & 9,1 \\
\hline \multicolumn{5}{|c|}{ \% códigos-basura (cáncer) * } \\
\hline Argentina & 1,4 & 1,3 & 1,3 & 1,3 \\
\hline GBA & 1,5 & 1,3 & 1,3 & 1,4 \\
\hline Pampeana & 1,5 & 1,5 & 1,2 & 1,4 \\
\hline Noroeste & 0,8 & 0,9 & 0,9 & 0,9 \\
\hline Noreste & 1,4 & 1,3 & 1,4 & 1,4 \\
\hline Cuyo & 1,1 & 1,1 & 1,0 & 1,1 \\
\hline Patagónica & 1,8 & 1,8 & 1,8 & 1,7 \\
\hline Ignorada & 0,9 & 1,4 & 0,7 & 1,0 \\
\hline \multicolumn{5}{|c|}{$\%$ causas poco útiles (total) ** } \\
\hline Argentina & 20,9 & 21,2 & 21,1 & 21,1 \\
\hline GBA & 23,6 & 23,6 & 23,1 & 23,4 \\
\hline Pampeana & 22,1 & 23,2 & 24,0 & 23,1 \\
\hline Noroeste & 17,9 & 16,1 & 15,9 & 16,6 \\
\hline Noreste & 18,4 & 18,7 & 16,9 & 18,0 \\
\hline Cuyo & 9,6 & 9,6 & 9,6 & 9,6 \\
\hline Patagónica & 13,7 & 14,1 & 14,8 & 14,2 \\
\hline Ignorada & 19,9 & 23,6 & 20,7 & 21,4 \\
\hline
\end{tabular}

GBA: Gran Buenos Aires.

* CIE10-causas mal definidas = códigos R00-R99; códigos basura lesiones = códigos Y10 a Y34, Y872; códigos basura enfermedades cardiovasculares = códigos 147.2, 149.0, 146, 150, 151.4, 151.5, 151.6, 151.9, 170.9; códigos basura cáncer = códigos C76, C80, C97;

** Causas poco útiles (total) = suma de mal definidas y códigos basura. 
El Grupo II, correspondiente a las enfermedades no transmisibles, abarcó el 82,9\% (varones: 81,8\%; mujeres: 84,1\%) de las causas de muerte en Argentina. Este grupo fue mayoritario en todas las regiones y ambos sexos, variando de 76,8\% en varones de la región Noroeste, a 85,7\% en mujeres de Cuyo. Las muertes por diabetes mellitus, pertenecientes al Grupo II, representaron alrededor del 3\% en todo el país, variando de 1,7\% en GBA a 6,5\% en Cuyo.

La proporción de muertes del Grupo I superó a la del Grupo III en prácticamente todas las regiones para el sexo masculino, excepto en Cuyo y Patagónica. Esta diferencia entre ambos grupos fue más acentuada en las mujeres en todas las regiones, con valores de 18,1\% para el Grupo I y 2,6\% para el III en mujeres del Noroeste (datos no tabulados).

Las tasas de mortalidad ajustadas por edad, para todos los grupos de causas, fueron más altas en varones respecto de las mujeres (Tabla 3). El Grupo II exhibió las tasas más elevadas de todos los grupos en ambos sexos, con un valor para Argentina de 15,3 por 1.000 habitantes en varones y 9,9 en mujeres. En este grupo, para ambos sexos, GBA fue la región con tasas más altas y la región Patagónica la de tasas más bajas, como también la de Noroeste para varones.

La tasa de mortalidad por diabetes mellitus ajustada por edad fue del 2,6\% y $3 \%$ de la tasa de mortalidad del Grupo II para Argentina en varones y mujeres respectivamente. Estos porcentajes fueron desde el 1,9\% para los varones de GBA a $8 \%$ para las mujeres de Cuyo.

Tabla 3

Tasas brutas y ajustadas de mortalidad (por 1.000 habitantes), según grupos de causas, diabetes mellitus y sexo. Argentina y regiones *, 2010.

\begin{tabular}{|c|c|c|c|c|c|c|c|c|}
\hline \multirow[t]{3}{*}{ Sexo/región } & \multicolumn{2}{|r|}{ Grupo I } & \multicolumn{4}{|c|}{ Grupo II } & \multicolumn{2}{|r|}{ Grupo III } \\
\hline & \multirow{2}{*}{$\begin{array}{l}\text { Tasa } \\
\text { bruta }\end{array}$} & \multirow{2}{*}{$\begin{array}{c}\text { Tasa ajustada ** } \\
\text { (IC95\%) }\end{array}$} & \multirow{2}{*}{$\begin{array}{l}\text { Tasa } \\
\text { bruta }\end{array}$} & \multirow{2}{*}{$\begin{array}{c}\text { Tasa ajustada ** } \\
\text { (IC95\%) }\end{array}$} & \multicolumn{2}{|c|}{ Diabetes mellitus } & \multirow{2}{*}{$\begin{array}{c}\text { Tasa } \\
\text { bruta }\end{array}$} & \multirow{2}{*}{$\begin{array}{l}\text { Tasa ajustada * } \\
\text { (IC95\%) }\end{array}$} \\
\hline & & & & & $\begin{array}{l}\text { Tasa } \\
\text { bruta }\end{array}$ & $\begin{array}{c}\text { Tasa ajustada ** } \\
\text { (IC95\%) }\end{array}$ & & \\
\hline \multicolumn{9}{|l|}{ Varones } \\
\hline Argentina & 1,9 & $2,3(2,3-2,3) * * *$ & 13,1 & $15,3(15,2-15,4)$ & 0,3 & $0,4(0,4-0,4) * * *$ & 1,0 & $1,0(1,0-1,0) * * *$ \\
\hline Cuyo & 1,3 & $1,6(1,5-1,7)$ & 12,2 & $14,5(14,2-14,8)$ & 0,9 & $1,0(1,0-1,1)$ & 1,1 & $1,2(1,1-1,3)$ \\
\hline GBA & 2,1 & $2,4(2,3-2,4)$ & 14,2 & $16,0(15,9-16,2)$ & 0,3 & $0,3(0,3-0,4)$ & 0,8 & $0,8(0,8-0,8) * \star *$ \\
\hline Noreste & 2,2 & $3,0(2,9-3,2)$ & 11,5 & $15,2(14,8-15,5)$ & 0,6 & $0,8(0,7-0,9)$ & 1,2 & $1,3(1,2-1,4)$ \\
\hline Noroeste & 2,2 & $3,0(2,9-3,1)$ & 10,8 & $14,0(13,7-14,3)$ & 0,5 & $0,7(0,6-0,7)$ & 1,1 & $1,1(1,0-1,2)$ \\
\hline Pampeana & 1,9 & $2,1(2,1-2,2)$ & 13,8 & $15,1(15,0-15,3)$ & 0,5 & $0,5(0,5-0,6)$ & 1,1 & $1,1(1,0-1,1)$ \\
\hline Patagónica & 1,1 & $1,8(1,7-2,0)$ & 9,4 & $14,0(13,5-14,4)$ & 0,5 & $0,8(0,7-0,9)$ & 1,0 & $1,1(1,0-1,2)$ \\
\hline \multicolumn{9}{|l|}{ Mujeres } \\
\hline Argentina & 1,8 & $1,6(1,5-1,6)$ & 11,3 & $9,9(9,8-9,9)$ & 0,3 & $0,3(0,3-0,3) * * *$ & 0,3 & $0,3(0,3-0,3) * * *$ \\
\hline Cuyo & 1,3 & $1,2(1,2-1,3)$ & 10,6 & $10,0(9,8-10,3)$ & 0,8 & $0,8(0,7-0,3)$ & 0,5 & $0,5(0,4-0,5)$ \\
\hline GBA & 2,0 & $1,6(1,6-1,6) * * *$ & 12,8 & $10,4(10,3-10,5)$ & 0,3 & $0,2(0,2-0,2) * * *$ & 0,3 & $0,2(0,2-0,2) * \star *$ \\
\hline Noreste & 1,8 & $2,0(1,9-2,1)$ & 8,8 & $10,0(9,8-10,3)$ & 0,7 & $0,7(0,7-0,8)$ & 0,4 & $0,4(0,3-0,4)$ \\
\hline Noroeste & 2,0 & $2,1(2,0-2,2)$ & 8,5 & $9,3(9,1-9,5)$ & 0,5 & $0,5(0,5-0,6)$ & 0,3 & $0,3(0,3-0,3) * * *$ \\
\hline Pampeana & 1,8 & $1,4(1,4-1,5)$ & 11,9 & $9,5(9,4-9,6)$ & 0,4 & $0,3(0,3-0,4)$ & 0,4 & $0,3(0,3-0,4)$ \\
\hline Patagónica & 1,1 & $1,4(1,2-1,5)$ & 7,0 & $8,7(8,4-9,0)$ & 0,4 & $0,5(0,5-0,6)$ & 0,2 & $0,3(0,2-0,3)$ \\
\hline
\end{tabular}

GBA: Gran Buenos Aires; Grupo I: enfermedades transmisibles, maternas, perinatales y nutricionales; Grupo II: enfermedades no transmisibles; Grupo III: lesiones; IC95\%: intervalo de confianza del 95\%.

* Gran Buenos Aires (Ciudad Autónoma de Buenos Aires -CABA- y 24 partidos del conurbano bonaerense); Pampeana (Provincia de Buenos Aires, excluyendo los 24 partidos del conurbano; Córdoba; La Pampa; Santa Fe; Entre Ríos); Noroeste (Catamarca; Jujuy; La Rioja; Salta; Santiago del Estero; Tucumán); Noreste (Corrientes; Chaco; Formosa; Misiones); Cuyo (Mendoza; San Juan; San Luis) y Patagónica (Chubut; Neuquén; Río Negro; Santa Cruz; Tierra del Fuego);

** Ajustada por edad y sexo, población patrón de Argentina del año 2010;

*** Las tasas redondeadas en una casilla decimal enmascaran pequeñas variaciones de intervalos de confianza.

Notas: (i) tasas calculadas con defunciones de personas de 30 años o más, expresadas por 1.000 habitantes; (ii) frecuencia de óbitos de 2010 , estimada a partir de la media de los óbitos ocurridos en el período 2009-2011. 
La carga representada por los AVPMP totales en población de Argentina de 30 años y más para 2010 fue de 3.730 .917 años (varones: 53,7\%; mujeres: 46,3\%) (Tabla 4). Para el mismo período, en términos medios, se perdieron 227,7 años de vida cada 1.000 varones y 154,1 años cada 1.000 mujeres.

En la Argentina y en todas las regiones, los varones perdieron más años de vida que las mujeres, independientemente de las causas.

Considerado el país, el 77,3\% de los AVPMP en varones se debieron a las enfermedades no transmisibles, $11,4 \%$ a las transmisibles y $11,3 \%$ a las lesiones. Estos porcentajes para las mujeres fueron de 83,4\%; 12,6\%; y 4\%, respectivamente. Las regiones que mostraron las tasas de AVPMP del Grupo II más elevadas fueron GBA en varones y Noroeste en mujeres (Tabla 4).

Del total de AVPMP, 57.935 años (2,9\%) en varones y 58.162 años $(3,4 \%)$ en mujeres se debieron a la diabetes mellitus. La tasa ajustada de AVPMP debido a diabetes mellitus que para el país en su conjunto fue de 6,6 por 1.000 habitantes en varones y 7,3 en mujeres respectivamente, en Cuyo fue de 12,7 por 1.000 en varones y de 10,5 en mujeres. Las tasas ajustadas de AVPMP por diabetes mellitus más bajas se observaron en la región GBA (Tabla 4).

La tasa de AVPMP por diabetes mellitus ajustada por edad fue del 3,7\% y 5,7\% de la tasa de AVPMP del Grupo II para Argentina, en varones y mujeres respectivamente. Estos porcentuales variaron desde el 2,2\% en los varones de GBA a 8,2\% en las mujeres de Cuyo (Tabla 4).

Las tasas ajustadas de AVPMP por diabetes mellitus fueron más elevadas, en ambos sexos y para todas las regiones, en la población sin cobertura de salud (Tabla 5).

En todas las regiones y para ambos sexos se observaron valores más elevados de AVPMP en la población con cobertura de salud, a partir de los 45 años. Esta situación fue diferente en la población sin cobertura de salud, en la cual el grupo de 30-44 años presentó valores altos, mayores aún que para grupos de edad más avanzados en algunas regiones. Alrededor del 70-75\% de los AVPMP en esta población corresponden a grupos etarios jóvenes, de 30-44 años y 45-59 años. Este traslado hacia edades más jóvenes en la población sin cobertura respecto de la que sí la tiene, se observó en ambos sexos y para todas las regiones.

Con escasas excepciones, concentradas principalmente en la franja de 60-69 años, en todas las regiones y en ambos sexos se observó que las tasas de AVPMP fueron mayores en la población sin cobertura de salud, respecto de la población con cobertura. Esta situación fue más evidente para la región Patagónica, en la cual la relación llegó a ser del doble para ciertas franjas etarias, y hasta del triple para los varones de 30-44 años (Tabla 6).

\section{Discusión}

La importancia de contar con datos de mortalidad de buena calidad, como soporte a la producción epidemiológica para la implementación de políticas en salud, ha sido ampliamente documentada. Variadas investigaciones en diversas partes del mundo han colocado su foco de interés en la cuestión de la calidad de los datos de mortalidad 25,32,33,34.

La calidad de los registros de mortalidad de Argentina fue insatisfactoria para el trienio bajo estudio, a expensas de la elevada proporción total de códigos poco útiles; de acuerdo con Mathers et al. 25, los países con baja calidad de registros tienen una proporción de códigos poco útiles por encima del $20 \%$.

Un trabajo que estudió esta proporción, considerando Argentina y otros tres países de América Latina para el período 2000-2011, encontró un porcentaje de códigos poco útiles de 15\% para México; 13\% para Chile y Colombia y 39\% para Argentina 33. El hecho de que el estudio haya utilizado una clasificación de códigos poco útiles distinta a la usada en nuestra investigación, podría explicar las diferencias en los porcentajes hallados.

Una de las estrategias metodológicas para minimizar la repercusión de la elevada proporción de códigos poco útiles sobre la calidad de los resultados finales en los análisis de mortalidad es la redistribución de causas mal definidas, de acuerdo a otras características previamente conocidas 27,35 . Esta estrategia fue utilizada en nuestra investigación, con el objetivo de reducir los datos de baja calidad.

Si bien las codificaciones "poco útiles" no afectan de manera predominante a la diabetes mellitus, sí lo hacen fundamentalmente con las enfermedades cardiovasculares. Estas enfermedades integran el Grupo II, el cual es el de mayor representación en la carga fatal de Argentina y el mundo 11,35,36,37. 
Tabla 4

Años de vida perdidos por muerte prematura y tasas ajustadas según sexo y grupos de causas. Argentina y regiones *, 2009-2011.

\begin{tabular}{|c|c|c|c|c|c|c|}
\hline \multirow[t]{3}{*}{ Sexo/Región } & \multicolumn{2}{|r|}{ Grupo I } & \multicolumn{4}{|c|}{ Grupo II } \\
\hline & \multirow[t]{2}{*}{ AVPMP } & \multirow[t]{2}{*}{ Tasa ajustada ** (IC95\%) } & \multirow[t]{2}{*}{ AVPMP } & \multirow{2}{*}{$\begin{array}{c}\text { Tasa ajustada ** } \\
\text { (IC95\%) }\end{array}$} & \multicolumn{2}{|c|}{ Diabetes mellitus } \\
\hline & & & & & AVPMP & $\begin{array}{c}\text { Tasa ajustada ** } \\
\text { (IC95\%) }\end{array}$ \\
\hline \multicolumn{7}{|l|}{ Varones } \\
\hline Argentina & 228.560 & $26,4(26,3-26,5)$ & 1.546 .999 & $177,5(177,2-177,8)$ & 57.935 & $6,6(6,5-6,6)$ \\
\hline Cuyo & 9.797 & $16,8(16,4-17,1)$ & 97.518 & $162,9(161,9-164,0)$ & 7.747 & $12,7(12,4-13,0)$ \\
\hline GBA & 83.223 & $28,3(28,1-28,5)$ & 548.856 & $187,7(187,2-188,2)$ & 11.959 & $4,1(4,0-4,1)$ \\
\hline Noreste & 22.252 & $34,2(33,7-34,7)$ & 122.330 & $183,9(182,9-185,0)$ & 6.833 & $10,2(9,9-10,4)$ \\
\hline Noroeste & 29.908 & $33,5(33,1-33,9)$ & 152.124 & $167,7(166,9-168,6)$ & 7.763 & $8,4(8,2-8,6)$ \\
\hline Pampeana & 75.868 & $23,9(23,7-24,0)$ & 561.157 & $173,7(173,3-174,2)$ & 20.344 & $6,2(6,1-6,3)$ \\
\hline Patagónica & 7.512 & $19,1(18,7-19,6)$ & 65.040 & $162,0(160,7-163,3)$ & 3.305 & $8,5(8,2-8,8)$ \\
\hline \multicolumn{7}{|l|}{ Mujeres } \\
\hline Argentina & 217.310 & $19,2(19,1-19,3)$ & 1.442 .373 & $128,4(128,2-128,6)$ & 58.162 & $7,3(7,0-7,5)$ \\
\hline Cuyo & 10.593 & $14,1(13,9-14,4)$ & 96.430 & $128,5(127,7-129,3)$ & 7.947 & $10,5(10,3-10,8)$ \\
\hline GBA & 82.528 & $20,5(20,4-20,7)$ & 541.666 & $134,8(134,4-135,1)$ & 12.350 & $3,1(3,1-3,2)$ \\
\hline Noreste & 19.629 & $26,0(25,6-26,3)$ & 106.837 & $141,3(140,4-142,1)$ & 7.744 & $10,4(10,2-10,7)$ \\
\hline Noroeste & 27.507 & $25,7(25,4-26,0)$ & 135.930 & $127,5(126,8-128,1)$ & 7.842 & $7,4(7,2-7,6)$ \\
\hline Pampeana & 70.357 & $16,5(16,3-16,6)$ & 511.313 & $121,4(121,1-121,7)$ & 19.547 & $4,7(4,6-4,7)$ \\
\hline Patagónica & 6.646 & $14,9(14,6-15,3)$ & 50.172 & $111,7(110,7-112,7)$ & 2.736 & $6,3(6,1-6,6)$ \\
\hline \multirow[t]{2}{*}{ Sexo/Región } & & Grupo III & & Total & & \\
\hline & AVPMP & $\begin{array}{c}\text { Tasa ajustada ** } \\
\text { (IC95\%) }\end{array}$ & AVPMP & $\begin{array}{c}\text { Tasa ajustada ** } \\
\text { (IC95\%) }\end{array}$ & & \\
\hline \multicolumn{7}{|l|}{ Varones } \\
\hline Argentina & 226.856 & $23,9(23,8-24,0)$ & 2.002 .415 & $227,7(227,4-228,1)$ & & \\
\hline Cuyo & 16.494 & $25,3(25,0-25,7)$ & 123.809 & $205,0(203,9-206,2)$ & & \\
\hline GBA & 60.418 & $19,2(19,0-19,3)$ & 692.497 & $235,2(234,6-235,7)$ & & \\
\hline Noreste & 23.408 & $30,2(29,8-30,6)$ & 167.990 & $248,3(247,1-249,6)$ & & \\
\hline Noroeste & 27.863 & $26,1(25,8-26,4)$ & 209.895 & $227,3(226,3-228,3)$ & & \\
\hline Pampeana & 85.693 & $25,6(25,5-25,8)$ & 722.718 & $223,2(222,7-223,8)$ & & \\
\hline Patagónica & 12.952 & $25,1(24,7-25,6)$ & 85.504 & $206,3(204,8-207,8)$ & & \\
\hline \multicolumn{7}{|l|}{ Mujeres } \\
\hline Argentina & 68.819 & $5,2(5,2-5,3)$ & 1.728 .502 & $154,1(153,9-154,4)$ & & \\
\hline Cuyo & 6.383 & $8,7(8,5-9,0)$ & 113.406 & $151,4(150,5-152,3)$ & & \\
\hline GBA & 17.992 & $4,9(4,9-5,0)$ & 642.186 & $160,2(159,8-160,6)$ & & \\
\hline Noreste & 6.499 & $8,0(7,8-8,2)$ & 132.965 & $175,3(174,3-176,2)$ & & \\
\hline Noroeste & 7.202 & $6,4(6,2-6,5)$ & 170.639 & $159,6(158,8-160,3)$ & & \\
\hline Pampeana & 27.529 & $7,3(7,2-7,4)$ & 609.199 & $145,2(144,8-145,5)$ & & \\
\hline Patagónica & 3.141 & $6,1(5,9-6,3)$ & 59.959 & $132,7(131,7-133,8)$ & & \\
\hline
\end{tabular}

AVPMP: años de vida perdidos por muerte prematura; GBA: Gran Buenos Aires; Grupo I: enfermedades transmisibles, maternas, perinatales y nutricionales; Grupo II: enfermedades no transmisibles; Grupo III: lesiones; IC95\%: intervalo de confianza del 95\%.

* Gran Buenos Aires (Ciudad Autónoma de Buenos Aires -CABA- y 24 partidos del conurbano bonaerense); Pampeana (Provincia de Buenos Aires, excluyendo los 24 partidos del conurbano; Córdoba; La Pampa; Santa Fe; Entre Ríos); Noroeste (Catamarca; Jujuy; La Rioja; Salta; Santiago del Estero; Tucumán); Noreste (Corrientes; Chaco; Formosa; Misiones); Cuyo (Mendoza; San Juan; San Luis) y Patagónica (Chubut; Neuquén; Río Negro; Santa Cruz; Tierra del Fuego);

** Ajustada por edad y sexo, población patrón de Argentina del año 2010.

Notas: (i) tasas calculadas con defunciones de personas de 30 años o más, expresadas por 1.000 habitantes; (ii) frecuencia de óbitos de 2010 , estimada a partir de la media de los óbitos ocurridos en el período 2009-2011. 
Tabla 5

Tasas brutas y ajustadas de años de vida perdidos por muerte prematura debido a diabetes mellitus, defunciones de 30 años y más, según sexo y cobertura de salud. Argentina y regiones *, 2009-2011.

\begin{tabular}{|c|c|c|c|c|}
\hline \multirow[t]{2}{*}{ Sexo/Región } & \multicolumn{2}{|c|}{ Con cobertura } & \multicolumn{2}{|c|}{ Sin cobertura } \\
\hline & Tasa bruta & $\begin{array}{c}\text { Tasa ajustada ** } \\
\text { (IC95\%) }\end{array}$ & Tasa bruta & $\begin{array}{c}\text { Tasa ajustada ** } \\
\text { (IC95\%) }\end{array}$ \\
\hline \multicolumn{5}{|l|}{ Varones } \\
\hline Argentina & 19,4 & $18,7(18,6-18,8)$ & 15,7 & $25,5(25,0-25,9)$ \\
\hline Cuyo & 16,1 & $15,1(14,7-15,4)$ & 11,0 & $22,8(20,5-25,4)$ \\
\hline GBA & 20,0 & $19,2(19,0-19,3)$ & 16,0 & $24,8(23,9-25,7)$ \\
\hline Noreste & 25,2 & $24,7(24,2-25,2)$ & 18,5 & $27,8(26,7-28,9)$ \\
\hline Noroeste & 24,2 & $23,9(23,6-24,3)$ & 17,9 & $27,7(26,6-28,7)$ \\
\hline Pampeana & 18,3 & $17,2(17,0-17,4)$ & 14,6 & $26,2(25,2-27,1)$ \\
\hline Patagónica & 11,5 & $14,0(13,6-14,5)$ & 15,4 & $25,1(23,4-26,9)$ \\
\hline \multicolumn{5}{|l|}{ Mujeres } \\
\hline Argentina & 25,3 & $22,0(21,9-22,1)$ & 10,4 & $18,2(17,8-18,5)$ \\
\hline Cuyo & 13,0 & $11,0(10,7-11,3)$ & 8,6 & $15,1(14,0-16,3)$ \\
\hline GBA & 16,1 & $13,8(13,7-13,9)$ & 9,8 & $15,8(15,3-16,4)$ \\
\hline Noreste & 20,8 & $18,8(18,5-19,2)$ & 15,0 & $24,8(23,7-25,8)$ \\
\hline Noroeste & 20,1 & $18,3(18,0-18,6)$ & 13,2 & $21,0(20,2-21,9)$ \\
\hline Pampeana & 15,0 & $12,5(12,4-12,6)$ & 8,2 & $17,3(16,6-18,0)$ \\
\hline Patagónica & 10,2 & $10,8(10,4-11-1)$ & 12,8 & $22,7(21,2-24,3)$ \\
\hline
\end{tabular}

GBA: Gran Buenos Aires; IC95\%: intervalo de confianza del 95\%.

* Gran Buenos Aires (Ciudad Autónoma de Buenos Aires -CABA- y 24 partidos del conurbano bonaerense); Pampeana (Provincia de Buenos Aires, excluyendo los 24 partidos del conurbano; Córdoba; La Pampa; Santa Fe; Entre Ríos); Noroeste (Catamarca; Jujuy; La Rioja; Salta; Santiago del Estero; Tucumán); Noreste (Corrientes; Chaco; Formosa; Misiones); Cuyo (Mendoza; San Juan; San Luis) y Patagónica (Chubut; Neuquén; Río Negro; Santa Cruz; Tierra del Fuego);

** Ajustada por edad y sexo, población patrón de Argentina del año 2010.

Notas: (i) tasas calculadas con defunciones de personas de 30 años o más, expresadas por 1.000 habitantes; (ii) frecuencia de óbitos de 2010 , estimada a partir de la media de los óbitos ocurridos en el período 2009-2011.

Se verificó el peso considerable de las enfermedades del Grupo II en la carga de mortalidad para Argentina, situación más marcada aún en las mujeres; el mayor porcentaje de carga fatal debido a lesiones en varones respecto de las mujeres, y la mayor participación de grupos etarios más avanzados en la carga fatal por diabetes mellitus en las mujeres, respecto de los varones. Tales resultados coinciden con los descriptos por Borruel et al. 36 en Argentina para el año 2005.

En relación con la diabetes mellitus, el análisis regional develó que Cuyo y Noreste fueron las regiones con tasas ajustadas de mortalidad y tasas ajustadas de AVPMP más elevadas. Vale decir, en las mismas regiones donde las tasas de muerte por diabetes mellitus fueron elevadas, también lo fue el riesgo de muerte prematura por esta patología.

En las regiones Noroeste y Noreste, que son a su vez las más pobres del país (INDEC. http://www. indec.gov.ar/indicadores-sociodemograficos.asp, accedido el 07/Abr/2015), los porcentajes de carga de mortalidad debidos al grupo de las enfermedades transmisibles, las tasas ajustadas de mortalidad y las tasas ajustadas de AVPMP, fueron mayores que para el resto de las regiones. Cuestión que hace pensar que en el interior del país se reproducen los patrones de comportamientos de los grandes grupos de causas observados entre países, de acuerdo a su nivel de ingresos (IHME. http://vizhub. healthdata.org/gbd-compare/, accedido el 16/Jul/2014) 38.

Las diferencias regionales para la carga de mortalidad por diabetes mellitus no parecen explicarse a partir de una mayor prevalencia. Por ejemplo el Noreste, segunda región en importancia para mor- 
Tabla 6

Años de vida perdidos por muerte prematura (número absoluto y tasas) por diabetes mellitus, según franja etaria, sexo y cobertura de salud. Argentina y regiones *, 2010.

\begin{tabular}{|c|c|c|c|c|c|c|c|c|c|c|}
\hline \multirow{2}{*}{$\begin{array}{l}\text { Sexo/Cobertura de salud/ } \\
\text { Región }\end{array}$} & \multicolumn{2}{|c|}{ 30-44 años } & \multicolumn{2}{|c|}{ 45-59 años } & \multicolumn{2}{|c|}{ 60-69 años } & \multicolumn{2}{|c|}{ 70-79 años } & \multicolumn{2}{|c|}{$80+$ años } \\
\hline & AVPMP & Tasa ** & AVPMP & Tasa ** & AVPMP & Tasa ** & AVPMP & Tasa ** & AVPMP & Tasa ** \\
\hline \multicolumn{11}{|l|}{ Varones sin cobertura } \\
\hline Argentina & 16.625 & 11,8 & 16.258 & 16,2 & 7.007 & 23,5 & 2.127 & 63,3 & 1.271 & 130,0 \\
\hline Cuyo & 580 & 5,6 & 999 & 13,8 & .453 & 20,0 & 126 & 63,6 & 50 & 152,0 \\
\hline GBA & 6.534 & 15,2 & 4.620 & 14,6 & 1.732 & 17,3 & 474 & 46,5 & 363 & 147,6 \\
\hline Noreste & 2.204 & 13,2 & 2.073 & 17,9 & 1.108 & 34,8 & 338 & 64,8 & 217 & 118,4 \\
\hline Noroeste & 2.281 & 10,9 & 2.672 & 21,3 & 1.202 & 33,7 & 387 & 68,7 & 212 & 110,0 \\
\hline Pampeana & 4.446 & 10,2 & 5.095 & 15,6 & 2.169 & 23,4 & 638 & 78,1 & 333 & 133,9 \\
\hline Patagónica & 580 & 8,9 & 799 & 17,4 & 343 & 23,6 & 164 & 69,0 & 96 & 129,4 \\
\hline \multicolumn{11}{|l|}{ Varones con cobertura } \\
\hline Argentina & 14.575 & 5,9 & 24.427 & 12,8 & 28.496 & 27,6 & 32.324 & 45,7 & 25.097 & 83,7 \\
\hline Cuyo & 541 & 3,3 & 1.274 & 10,4 & 1.717 & 23,9 & 1.942 & 39,8 & 1.366 & 67,9 \\
\hline GBA & 6.070 & 7,0 & 8.567 & 13,6 & 9.800 & 28,3 & 10.852 & 44,7 & 8.811 & 79,2 \\
\hline Noreste & 1.082 & 6,9 & 2.423 & 17,8 & 2.450 & 37,3 & 2.918 & 62,4 & 1.779 & 105,6 \\
\hline Noroeste & 1.740 & 7,0 & 3.172 & 16,1 & 3.870 & 38,4 & 3.962 & 59,8 & 2.692 & 102,1 \\
\hline Pampeana & 4.717 & 5,3 & 8.092 & 11,4 & 9.816 & 24,3 & 11.635 & 42,0 & 9.693 & 83,6 \\
\hline Patagónica & 425 & 2,8 & 899 & 8,2 & 843 & 18,6 & 1.015 & 39,4 & 756 & 81,8 \\
\hline \multicolumn{11}{|l|}{ Mujeres sin cobertura } \\
\hline Argentina & 11.844 & 8,4 & 8.375 & 8,9 & 2.904 & 19,0 & 1.828 & 48,9 & 1.749 & 96,2 \\
\hline Cuyo & 663 & 6,3 & 572 & 7,8 & 255 & 22,4 & 98 & 41,5 & 71 & 61,2 \\
\hline GBA & 4.419 & 9,9 & 2.350 & 7,1 & 608 & 10,2 & 402 & 31,6 & 634 & 105,3 \\
\hline Noreste & 2.033 & 12,3 & 1.115 & 11,2 & 628 & 37,8 & 341 & 66,0 & 228 & 96,2 \\
\hline Noroeste & 2.033 & 9,7 & 1.476 & 13,3 & 510 & 26,7 & 353 & 57,1 & 228 & 78,7 \\
\hline Pampeana & 2.210 & 5,2 & 2.440 & 8,4 & 707 & 18,4 & 488 & 56,1 & 441 & 96,5 \\
\hline Patagónica & 486 & 8,1 & 422 & 10,9 & 196 & 25,8 & 146 & 64,8 & 147 & 127,4 \\
\hline \multicolumn{11}{|l|}{ Mujeres con cobertura } \\
\hline Argentina & 14.185 & 8,2 & 19.585 & 14,2 & 26.203 & 30,5 & 34.410 & 56,8 & 41.954 & 115,1 \\
\hline Cuyo & 663 & 3,7 & 1.115 & 7,7 & 1.649 & 16,7 & 2.328 & 35,2 & 2.227 & 60,4 \\
\hline GBA & 5.789 & 6,3 & 7.412 & 10,1 & 9.284 & 18,9 & 11.592 & 32,0 & 15.757 & 67,0 \\
\hline Noreste & 1.237 & 6,8 & 1.989 & 12,7 & 2.355 & 27,2 & 2.986 & 50,8 & 2.440 & 82,8 \\
\hline Noroeste & 1.768 & 6,4 & 2.742 & 11,9 & 3.533 & 27,2 & 4.498 & 52,2 & 3.942 & 85,8 \\
\hline Pampeana & 4.198 & 4,4 & 5.604 & 7,0 & 8.675 & 16,2 & 11.872 & 30,5 & 16.462 & 69,1 \\
\hline Patagónica & 530 & 3,2 & 723 & 6,1 & 707 & 13,2 & 1.134 & 35,3 & 1.126 & 65,8 \\
\hline
\end{tabular}

AVPMP: años de vida perdidos por muerte prematura; GBA: Gran Buenos Aires.

* Gran Buenos Aires (Ciudad Autónoma de Buenos Aires -CABA- y 24 partidos del conurbano bonaerense); Pampeana (Provincia de Buenos Aires, excluyendo los 24 partidos del conurbano; Córdoba; La Pampa; Santa Fe; Entre Ríos); Noroeste (Catamarca; Jujuy; La Rioja; Salta; Santiago del Estero; Tucumán); Noreste (Corrientes; Chaco; Formosa; Misiones); Cuyo (Mendoza; San Juan; San Luis) y Patagónica (Chubut; Neuquén; Río Negro; Santa Cruz; Tierra del Fuego);

** Tasa de AVPMP por edad y sexo, población patrón de Argentina del año 2010.

Notas: (i) tasas calculadas con defunciones de personas de 30 años o más, expresadas por 1.000 habitantes; (ii) frecuencia de óbitos de 2010, estimada a partir de la media de los óbitos ocurridos en el período 2009-2011.

talidad y carga fatal por diabetes mellitus, fue la que registró la menor prevalencia de diabetes mellitus para el año 20099 entre todas las regiones, aunque las diferencias no fueron significativas.

En nuestro estudio las tasas ajustadas de AVPMP fueron mayores en las regiones más pobres. Estos resultados corroboran los hallazgos del segundo estudio de carga de enfermedad en Brasil, aunque las tasas se calcularon para la población en su conjunto, y no sólo para las personas de edad mayor 
de 30 años 35 . En ambos trabajos se destacó la preponderancia de las enfermedades del Grupo II en todas las regiones y tasas ajustadas de AVPMP mayores en varones, respecto de las mujeres.

En el estudio sobre carga de enfermedad a nivel nacional y subnacional de Australia ${ }^{37}$, se evidenció una mayor representación de los varones en la carga fatal y una notoria preeminencia de las enfermedades crónicas no transmisibles, al igual que en nuestro trabajo, así como un aumento en la tasa de AVPMP, a medida que se avanza en edad. En la presente investigación se evidenció dicho patrón para los Grupos I y II, no así para el Grupo III en que los varones jóvenes presentaron tasas elevadas.

Respecto del Grupo III, en nuestro trabajo, así como en el de Brasil 35 y el de Australia 37, fue marcada la diferencia entre sexos, respecto del porcentaje de la carga de mortalidad debida a lesiones a favor de los varones, fundamentalmente en los grupos etarios jóvenes.

Encontramos valores más elevados de tasas ajustadas de mortalidad y tasas ajustadas de AVPMP en varones en relación con las mujeres para todos los grupos de causas, sólo se constató una excepción en la tasa ajustada de AVPMP por diabetes mellitus que fue más elevada para las mujeres en Argentina. Cabe pensar que en estas diferencias por sexo, especialmente en el grupo de las enfermedades crónicas que requieren de seguimiento y consultas frecuentes a los efectores de salud, podría estar operando el ya conocido comportamiento femenino, en procura de servicios para su atención, de forma más temprana y frecuente 39 .

Para el caso particular de la diabetes mellitus, su prevalencia en Argentina para el año 2009 fue más elevada en las mujeres que en los varones. La prevalencia de inactividad física, uno de los principales factores involucrados en el desarrollo de la enfermedad y su abordaje terapéutico, fue más alta en mujeres ${ }^{9}$. Estos elementos pueden haber contribuido para explicar la observación de la tasa ajustada de AVPMP por diabetes mellitus más elevada en mujeres.

Fueron llamativas las diferencias que hallamos en relación con la pertenencia a algún sistema de cobertura de la salud para los resultados de mortalidad por diabetes mellitus, así como el traslado de la muerte prematura por diabetes mellitus a edades más jóvenes en la población sin cobertura de salud. La prevalencia de diabetes mellitus en este grupo también ha sido reportada como mayor respecto del que sí posee cobertura de salud 9 . Otro factor que puede haber operado para explicar el diferencial de tasas según cobertura de salud, es la posibilidad de realizar el tratamiento correspondiente: para el año 2013, el tratamiento para la diabetes mellitus resultó más frecuente en las personas con cobertura de salud 10 .

En un trabajo realizado en el año 2011 por Gakidou et al. 40, en relación con 7 países del mundo, se analizaron determinantes para el diagnóstico y tratamiento oportuno de la diabetes; la posibilidad de ser diagnosticado de diabetes mellitus, portando la enfermedad, estuvo asociada significativamente a la pertenencia a algún sistema de cobertura de salud. Al demostrarse que el acceso económico al tratamiento fue un factor de previsibilidad importante para el diagnóstico y tratamiento de la enfermedad, es muy probable que el hecho de contar con algún tipo de cobertura de salud en nuestro país haya incidido favorablemente en la posibilidad de ser abordado oportunamente y de evitar una muerte prematura.

La brecha entre las tasas de la población sin cobertura de salud versus con cobertura en la región Patagónica es llamativa. La encuesta de acceso a la salud en Argentina realizada en 201039 reveló que la región Patagónica, junto con el Noreste, fueron las regiones con menor porcentaje de población consultante al médico en los treinta días previos a la entrevista, situación que también se observó en los pueblos y zonas rurales respecto de las ciudades.

Por otro lado, dos trabajos sobre accesibilidad geográfica en el primer nivel de atención realizados en el país 41,42, llamaron la atención sobre la distancia al efector de salud y problemas con el transporte público, como factores que dificultarían la accesibilidad a los servicios públicos en dicho nivel. Si bien los trabajos fueron realizados en zonas pertenecientes a regiones diferentes de la Patagónica, la información sobre el bajo porcentaje de población consultante allí podría estar relacionada con barreras del tipo de las descritas, dado además que se trata de la región menos densamente poblada del país 43 . Esta cuestión merecería el desarrollo de estudios específicos en la región.

Otro aspecto a considerar es la fragmentación del sistema de salud de Argentina en tres subsectores, privado, público y obras sociales, con fuentes de financiamiento, red institucional y lógicas diferentes 23,44 . Esto hace pensar que podría estar operando una gran heterogeneidad en el funcionamiento de los mismos en las diferentes regiones del país. 
Un análisis en profundidad de los sistemas de atención de la diabetes mellitus por regiones, posiblemente, contribuiría a explicar algunas de las diferencias observadas en nuestro trabajo. El hecho de que las dos regiones más pobres del país (Noroeste y Noreste) exhiban las tasas ajustadas de muerte prematura por diabetes mellitus más elevadas en la población sin cobertura, orienta a considerar que múltiples aspectos relacionados con el desarrollo, evolución y asistencia de la enfermedad pudieran estar vulnerados allí.

Una revisión reciente realizada en Argentina llevó a cabo una sistematización del tratamiento de la diabetes mellitus en la normativa sanitaria del país 45. Se observó que las provincias fueron incorporando, desde el punto de vista normativo e institucional, aspectos ligados a la prevención y control de la enfermedad en diferentes momentos temporales. Esto hace que las provincias se encuentren en distintos estadios de avance, respecto al marco normativo y legal que regula los programas destinados al abordaje de la diabetes mellitus, y que sostiene, por ejemplo, la provisión de fármacos para el tratamiento de la enfermedad.

Las diferencias regionales observadas en el trabajo respecto de la carga de mortalidad sugieren la necesidad de profundizar en líneas de investigación desde la óptica de las desigualdades en diabetes mellitus y sus determinantes en Argentina, que pudieran contribuir para la comprensión y abordaje de tales diferencias. Lo mismo debe pensarse, de manera general, para las ECNT, grupo con la mayor expresión en la carga de mortalidad en Argentina.

Esta necesidad se ve reforzada por las evidencias surgidas, a partir del informe del grupo del Global Burden of Disease (GBD) para Argentina para el 2010 (IHME. http://www.healthdata.org/print/4243, accedido el 15/Jul/2014), del cual surge que la diabetes mellitus fue una de las enfermedades que mostró un aumento del número absoluto de AVPMP entre 1990 y 2010, así como del porcentaje de la carga fatal total debida a diabetes mellitus, que pasó del 2\% al 2,5\%. Por otro lado, un informe para 240 países reveló que la diabetes mellitus fue una de las enfermedades que incrementó su tasa de muerte ajustada por edad entre 1990 y 201338.

La utilización de una tabla de vida propia para Argentina, considerando la mortalidad observada en las diferentes regiones, permitió obtener información sobre carga de mortalidad en un contexto más cercano a la situación contemporánea de esperanza de vida en el país. La tabla de vida propuesta internacionalmente 24 se basa en la esperanza de vida estimada para una mujer en Japón y Corea para el año 2050, escenario que dista de las condiciones actuales para Argentina.

Algunas diferencias metodológicas con el estudio de carga de Argentina 36 fueron que realizó ajustes por la tasa de descuento y tuvo en cuenta el valor social de la edad de la muerte. Además, los cálculos incluyeron todos los grupos etarios y no sólo la población de 30 años y más. Estas cuestiones nos imponen limitaciones para realizar comparaciones con nuestros resultados, particularmente en términos de cuantía de AVPMP.

Una limitación importante de la investigación está relacionada con la calidad de las fuentes de datos de mortalidad en Argentina. Cabe la posibilidad de que las diferencias regionales en las tasas de muerte prematura observadas en nuestro trabajo, fundamentalmente las asociadas al grupo de enfermedades no transmisibles y en menor medida las correspondientes a diabetes mellitus, podrían presentar variaciones si contásemos con proporciones mínimas de causas mal definidas.

La construcción de la tabla teórica para Argentina y los cuidados metodológicos tales como la redistribución de códigos poco útiles, así como el escaso impacto de los códigos basura sobre la diabetes mellitus, constituyeron esfuerzos en pos de la obtención de resultados válidos.

\section{Consideraciones finales}

La calidad de los datos tiene implicaciones directas en el potencial de los estudios de mortalidad como sustento de políticas públicas. Surge la necesidad de invertir en esfuerzos continuos en pos de la mejoría de la calidad de la codificación de la causa básica de muerte en Argentina.

Uno de los principales aportes del presente trabajo fue la posibilidad de obtener información sobre carga de mortalidad actualizada para Argentina y con nivel de agregación subnacional, por grandes grupos de causas y para diabetes mellitus en particular. Se sugiere considerar la utilización de 
la tabla de vida teórica para Argentina en futuros estudios de carga de enfermedad en el país, lo cual permitiría realizar comparaciones.

Se espera que el efecto beneficioso de la pertenencia a algún sistema de cobertura de la salud sobre la mortalidad prematura por diabetes mellitus represente un elemento más que contribuya a reforzar las acciones orientadas a universalizar la cobertura preventiva, de diagnóstico y tratamiento de la enfermedad, con especial énfasis en las regiones más pobres y que ostentan menor porcentaje de población con cobertura de salud.

\section{Colaboradores}

Todos los autores participaron de la concepción del proyecto, el análisis e interpretación de los datos, la redacción del artículo, la revisión crítica del contenido intelectual, y la aprobación final de la versión a ser publicada.

\section{Agradecimientos}

Agradecemos a la Administración Nacional de Laboratorios e Institutos de Salud (ANLIS), y al Instituto Nacional de Epidemiología Dr. Juan H. Jara de Argentina, por el apoyo económico e institucional recibido para la realización de la Maestría en Epidemiología en Salud Pública, en el marco del convenio ANLIS-Fiocruz por parte de uno de los autores. Este artículo es parte del trabajo realizado en la tesis final de la Maestría, perteneciente a la segunda cohorte. Agradecemos a la Dra. Alicia Lawrynowicz por la bibliografía aportada, así como por su apoyo para el desarrollo de la temática. Agradecemos a la Lic. Emilia Pepa, por la lectura de la versión final del artículo y sus valiosos aportes, orientados a mejorar la comprensión de lo escrito.

\section{Referencias}

1. International Diabetes Federation. IDF diabetes atlas. 7th Ed. Brussels: International Diabetes Federation; 2015.

2. Caporale JE, Elgart JF, Gagliardino JJ. Diabetes in Argentina: costs and managment of diabetes and its complications and challenges for health policy. Glob Health 2013; 9:1-10.

3. Ledón LLanes L. Impacto psicosocial de la diabetes mellitus, experiencias, significados y respuestas a la enfermedad. Rev Cubana Endocrinol 2012; 23:76-97.

4. Robles González E, Mestre JB, Benavides FG. La transición sanitaria: una revisión conceptual. Boletín de la Asociación de Demografía Histórica 1996; XVI:117-44.

5. Ortiz Z. Enfermedades crónicas no transmisibles. Buenos Aires: Ministerio de Salud de la Nación; 2005.

6. Barría PRM, Amigo CH. Transición nutricional: una revisión del perfil latinoamericano. Arch Lationam Nutr 2006; 56:3-11.

7. Organización Mundial de la Salud. Informe sobre la salud en el mundo 2003: forjemos el futuro. Geneva: Organización Mundial de la Salud; 2003.

8. Popkin BM. The nutrition transition and obesity in the developing world. J Nutr 2001; 131 Suppl 3:871s-3.

9. Ministerio de Salud de la Nación; Instituto Nacional de Estadísticas y Censos. Segunda Encuesta Nacional de Factores de Riesgo para Enfermedades No Transmisibles. Buenos Aires: Ministerio de Salud de la Nación; 2011.

10. Ministerio de Salud de la Nación; Instituto Nacional de Estadísticas y Censos. Tercera Encuesta Nacional de Factores de Riesgo para Enfermedades No Transmisibles. Buenos Aires: Ministerio de Salud de la Nación; 2015.

11. Dirección de Estadísticas e Información en Salud, Ministerio de Salud de la Nación. Estadísticas vitales-información básica año 2014. Buenos Aires: Ministerio de Salud de la Nación; 2015. 
12. Reichenheim ME, Werneck GL. Anos potenciais de vida perdidos no Rio de Janeiro, 1990. As mortes violentas em questão. Cad Saúde Pública 1994; 10 Suppl 1:188-98.

13. Murray CJL, López AD. The global burden of disease: a comprehensive assessment of mortality and disability from diseases, injuries, and risk factors in 1990 and projected to 2020. Cambridge: Harvard University Press; 1996.

14. Alleyne GA, Castillo-Salgado C, Schneider C, Mujica OJ, Loyola E, Vidaurre M. La medición de las desigualdades en salud: algunos ejemplos de la región de las Américas. In: Equity \& health: views from the Pan American Sanitary Bureau. Washington DC: Pan American Health Organization; 2001. p. 69-75. (Occasional Publication, 8).

15. Ministério da Saúde. Saúde Brasil 2006: uma análise da desigualdade em saúde. Brasilia: Ministério da Saúde; 2006.

16. Paim JS, Silva LMV, Costa MCN, Prata PR, Lessa I. Desigualdades na situação de saúde do município de Salvador e relações com as condições de vida. Rev Ciênc Méd Biol 2003; 2:309.

17. Victora C, Barros F, Vaughan JP. Epidemiología de la desigualdad. Washington DC: Organización Panamericana de la Salud; 1992.

18. Almeida Filho N. A problemática teórica da determinação social da saúde. In: Nogueira RP, organizador. Determinação social da saúde e reforma sanitária. Rio de Janeiro: Centro Brasileiro de Estudos de Saúde; 2010. p. 13-36.

19. Kawachi I, Subramanian SV, Almeida Filho N. A glossary for health inequalities. J Epidemiol Community Health 2002; 56:647-52.

20. Danaei G, Friedman AB, Oza S, Murray CJL, Ezzati M. Diabetes prevalence and diagnosis in US states: analysis of health surveys. Popul Health Metr 2009; 7:1-13.

21. Instituto Nacional de Estadísticas y Censos. Censo nacional de población, hogares y viviendas 2010: censo del bicentenario. Resultados definitivos. Serie B no 2, Tomo 2. Buenos Aires: Instituto Nacional de Estadísticas y Censos; 2012.

22. Organización Panamericana de la Salud. Clasificación estadística internacional de enfermedades y problemas relacionados con la salud, décima revisión. v. 1. Washington DC: Organización Panamericana de la Salud; 2003. (Publicación Científica, 554).

23. Belmartino S. Una década de reforma de la atención médica argentina. Salud Colect 2005; 1:155-71.

24. World Health Organization. WHO methods and data sources for global burden of disease estimates 2000-2011. Global Health Estimates Technical Paper. Geneva: World Health Organization; 2013.

25. Mathers CD, Fat DM, Inoue M, Rao CH, López A. Counting the dead and what they died from: an assessment of the global status of cause of death data. Bull World Health Organ 2005; 83:171-7.
26. Organización Panamericana de la Salud. Indicadores básicos 2013. Washington DC: Organización Panamericana de la Salud; 2013.

27. Gadelha AMJ, Leite IC, Valente JG, Schramm JMA, Portela MC, Campos MR. Relatório final do Projeto Estimativa da Carga de Doença do Brasil - 1998. Rio de Janeiro: Escola Nacional de Saúde Pública, Fundação Oswaldo Cruz; 2002.

28. World Health Organization. National burden of disease studies: a practical guide. Edition 2.0. Geneva: World Health Organization; 2001.

29. Voss T. Life table, life expectancy and healthadjusted life expectancy [HALE]. Seattle: Institute for Health Metric and Evaluation/University of Washington; 2014.

30. Murray CJL, Ezzati M, Flaxman AD, Lim S, Lozano R, Michaud C, et al. GBD 2010: design, definitions, and metrics. Lancet 2012; 380:2063-6.

31. Ministerio de Salud de la Nación. Guía para investigaciones en salud humana. Régimen de buena práctica clínica para estudios de farmacología clínica. Buenos Aires: Ministerio de Salud de la Nación; 2011.

32. Alazraqui M, Spinelli H, Zunino MG, Souza Ramos E. Calidad de los sistemas de información de mortalidad por violencias en Argentina y Brasil-1990-2010. Ciênc Saúde Coletiva 2012; 17:3279-87.

33. Ribotta SB, Escanés G. Códigos poco útiles en los registros de defunciones en Argentina, Chile, Colombia y México (2000-2011). Revista Electrónica Medicina, Salud y Sociedad 2014; 5:4-17.

34. World Health Organization. WHO methods and data sources for country-level causes of death 2000-2012. Global Health Estimates Technical Paper. Geneva: World Health Organization; 2014.

35. Leite IC, Valente JG, Mendes SJA. Carga de Doença do Brasil, 2008. Relatório final do projeto. Rio de Janeiro: Escola Nacional de Saúde Pública Sergio Arouca, Fundação Oswaldo Cruz; 2013

36. Borruel MA, Mas IP, Borruel GD. Estudio de carga de enfermedad: Argentina. Buenos Aires: Ministerio de Salud de la Nación; 2010.

37. Begg S, Voss T, Barker B, Stevenson C, Stanley L, López AD. The burden of disease and injury in Australia 2003. Canberra: Australian Institute of Health and Welfare; 2007.

38. GBD 2013 Mortality and Causes of Death Collaborators. Global, regional, and national age-sex specific all-cause and cause-specific mortality for 240 causes of death, 1990-2013: a systematic analysis for the Global Burden of Disease Study 2013. Lancet 2015; 385:117-71.

39. Marconi E, Fernández MM, Guevel C, Jorrat JR. El acceso a la salud en Argentina. III Encuesta de utilización y gasto en servicios de salud-2010. Buenos Aires: Ministerio de Salud de la Nación; 2011. 
40. Gakidou E, Mallinger L, Abbott-Klafter J, Guerrero R, Villalpando S, Ridaura RL, et al. Management of diabetes and associated cardiovascular risk factors in seven countries: a comparison of data from national health examination surveys. Bull World Health Organ 2011; 89:172-83.

41. Ponce BE. Análisis de la accesibilidad geográfica en la atención primaria de la salud: estudio aplicado en los centros regionales de referencias del Gran Resistencia (Chaco, Argentina). Rev Salud Pública 2015; 19:22-44.

42. De Petri D, Dietrich P, Mayo P, Carcagno A, De Titto E. Indicadores de accesibilidad geográfica a los centros de atención primaria para la gestión de inequidades. Rev Panam Salud Pública $2012 ; 34: 452-60$.

\begin{abstract}
The aim of this study was to estimate the mortality burden according to major groups of causes, with a focus on diabetes mellitus, as well as differences according to the presence or absence of health coverage for Argentina as a whole and its regions. The study used the mortality database for individuals 30 years or older in 2009-2011, stratified by sex, age bracket, region, underlying cause of death, and health coverage. We calculated the mortality rates and years of life lost due to premature mortality (YLLs). The quality of mortality records in Argentina was unsatisfactory, due to the high proportion of garbage codes for deaths. The main results showed a preponderance of chronic non-communicable diseases in the mortality burden in all regions of Argentina. In the Northeast, one of the country's poorest regions, the burden of mortality was high for all groups of causes. The mortality rates and adjusted YLL rates for diabetes mellitus were highest in the Cuyo region. There were marked differences in adjusted YLL rates according to health coverage status. The study's results highlight the need to develop measures to decrease regional and socioeconomic inequalities identified by mortality differences in Argentina.
\end{abstract}

Diabetes Mellitus; Health Status Disparities; Mortality
43. Instituto Nacional de Estadísticas y Censos. Censo nacional de población, hogares y viviendas 2010: censo del bicentenario. Resultados definitivos. Serie B no 2, Tomo 1. Buenos Aires: Instituto Nacional de Estadísticas y Censos; 2012.

44. Belmartino S. La atención médica argentina en el siglo XX. Buenos Aires: Siglo XXI Editores; 2005.

45. Lawrynowicz A, Alonso V. El tratamiento de la diabetes mellitus en la normativa sanitaria argentina: una sistematización. Rev Argent Salud Pública 2013; 4:39-44.

\section{Resumo}

O estudo teve como objetivo avaliar a carga de mortalidade por grandes grupos de causas, com foco na diabetes mellitus, assim como seus diferenciais, segundo presença ou ausência da cobertura por algum sistema de saúde, para Argentina e suas regiões. $O$ estudo foi realizado baseado nos óbitos de indivíduos de 30 anos adiante, acontecidos no triênio 2009-2011, estratificados segundo o sexo, faixa etária, região, causa básica de morte e cobertura de saúde. Foram calculadas taxas de mortalidade e de anos de vida perdidos por morte prematura (AVPMP). A qualidade dos registros relacionados à mortalidade na Argentina foi insatisfatória, devida à elevada proporção de códigos de causas de morte pouco úteis. Os principais resultados evidenciaram a preponderância do grupo de doenças crônicas na carga de mortalidade em todas as regiões do país. No Nordeste, uma das regiões mais pobres do país, a carga de mortalidade foi elevada para todos os grupos de causas. As taxas de mortalidade e de AVPMP por diabetes mellitus ajustadas foram mais elevadas na região de Cuyo. Destaca-se a diferença marcada nas taxas ajustadas de AVPMP, segundo a situação da cobertura de saúde. Este estudo norteia o rumo do desenvolvimento de ações que visam diminuir as inequidades regionais e socioeconômicas evidenciadas nos diferenciais de mortalidade na Argentina.

Diabetes Mellitus; Disparidades nos Níveis de Saúde; Mortalidade

Recibido el 01/Jul/2016

Versión final presentada el 01/Nov/2016

Aprobado el 06/Ene/2017 
Marro MJ et al. 